\title{
Arbor
}

\section{Alteraciones del relato: los niños con TDAH}

\author{
Amparo Ygual Fernández, Ana Miranda Casas
}

Arbor CLXVII, 697 (Enero 2004), 189-203 pp.

Los niños con TDAH tienen dificultades para la elaboración y comprensión de relatos relacionadas con las características cognitivas propias del trastorno. Las alteraciones que experimentan en el control inhibitorio, en la memoria de trabajo $y$, en suma, en el funcionamiento ejecutivo son responsables de los problemas en el relato. Estos se pueden producir en varios estadios del procesamiento: las dificultades atencionales pueden influir interfiriendo en la entrada de la información; las dificultades en la memoria operativa los hace menos eficaces en el reparto y control de recursos durante la realización de la tarea, lo cual se traduce en dificultades en la evocación de sucesos, más errores en el establecimiento de conexiones causales de los mismos, todo ello interferido por la no desactivación de información irrelevante para la narración. La memoria verbal, concretamente, es un elemento clave en las dificultades en funciones ejecutivas de los niños con TDAH. Por último, también se ha observado en el discurso problemas en la selección y manejo de las estructuras lingüisticas de base produciendo más errores de cohesión.

Estas dificultades en el relato conllevan numerosas implicaciones en la vida diaria de tipo social y académico. Implicaciones en la capacidad representacional, en las estrategias de solución de problemas, en la habilidad para interpretar las acciones de los otros y para realizar conexiones entre sus propias acciones y las consecuencias de las mismas.

El relato es una tarea cognitivamente compleja que requiere conjugar habilidades lingüísticas y cognitivas con un sobreesfuerzo organizativo al 
servicio de la información que se quiere transmitir. Esto supone realizar tareas cognitivas de primer orden relacionadas con una fuerte exigencia en organización y planificación, es decir, en función ejecutiva. Las alteraciones en la función ejecutiva pueden provocar, entre otras consecuencias, alteraciones en el lenguaje, que son especialmente evidentes en el relato. Este es el caso de los niños con Trastorno por Déficit de Atención con Hiperactividad (TDAH).

\section{Características cognitivas del TDAH}

El TDAH es un trastorno neurológico de inicio en la infancia. Se trata de una patología crónica cuyos síntomas, aunque cambiantes, permanecen a lo largo del tiempo, y se manifiestan en los distintos contextos en los que el niño y, más tarde, el adulto, se desenvuelve. Interfiere en la vida cotidiana, en los aprendizajes escolares, en el trabajo y también en las relaciones sociales de las personas que lo padecen. Su prevalencia en la población es alta: $6 \%$ en la edad infantil y un $4 \%$ en la vida adulta.

El Trastorno por Déficit de Atención con Hiperactividad se caracteriza por niveles inapropiados de atención, de hiperactividad y de impulsividad, según los criterios diagnósticos que se especifican en el DSM IVTR (American Psyquiatric Asociation, 2000). Los problemas de atención afectan a dominios como vigilancia, atención dividida, atención sostenida y atención selectiva. La impulsividad se caracteriza por una rapidez excesiva en el procesamiento de la información, un fracaso en esperar a emitir una respuesta hasta que se haya reunido información suficiente e inhibir mecanismos que resultan inapropiados para conseguir la meta. Goldstein (1999) considera que la definición práctica del TDAH incluye cinco componentes, valorando la impulsividad como el elemento decisivo en el moldeamiento de los otros cuatro componentes restantes, inatención, excesivo arousal, dificultad con la gratificación, en la expresión de emociones y locus de control. Sin embargo tal vez sean las manifestaciones de hiperactividad las que más diferencian a los escolares con TDAH de sus compañeros, tales como actividad motora gruesa, pequeños movimientos, interferir con otros y estar fuera del asiento. Los niños hiperactivos, incluso, tienen niveles superiores de movimiento durante el sueño. Hay que destacar, sin embargo, que este excesivo movimiento corporal va disminuyendo a lo largo de la niñez de tal forma que a partir de los 12 años las diferencias en este aspecto entre escolares con TDAH y normales se minimizan. En la adolescencia incluso adopta generalmente la forma de sentimientos de inquietud y dificultades para dedicarse a activi- 
dades sedentarias o tranquilas. Las dificultades en estos tres dominios se pueden detectar con claridad entre los 6 y 9 años.

En base a estos síntomas, según predomine un grupo de ellos u otro, el DSM-IV-TR distingue tres subtipos diferenciados del trastorno: El subtipo de TDAH predominantemente inatento (TDAH/I) es aquel en el que predominan los síntomas de baja atención, en el subtipo hiperactivoimpulsivo (TDAH/H-I) predominan los síntomas de hiperactividad e impulsividad, mientras que en el subtipo combinado (TDAH/C) se pueden distinguir los tres grupos de síntomas. Los subtipos más frecuentes son el TDAH combinado y el inatento.

Sólo alrededor de un 50\% de niños con TDAH sufren dificultades importantes en la adquisición de los niveles básicos del lenguaje, lo que hace suponer que ésta es una condición añadida al trastorno, que viene a agravar las dificultades en el desarrollo cognitivo y social y a acrecentar los problemas para el aprendizaje. Sin embargo, parece que ciertas peculiaridades en la pragmática lingüística están presentes en todos ellos. Tal y como se recoge en el DSM-IV-TR, en los niños con TDAH en edad escolar pueden presentarse síntomas como contestaciones impulsivas e inadecuadas en clase, dificultades para mantener el turno conversacional y habla excesiva como signos de su hiperactividad e impulsividad. Como síntomas de inatención se menciona el que aparentemente parece que no escuchan, que con frecuencia olvidan o tienen dificultades para seguir instrucciones, síntomas que se relacionan con dificultades de comprensión. En suma, todos ellos representan aspectos en el funcionamiento lingüístico que pueden ser inherentes al TDAH y que no se consideran un trastorno comórbido.

Durante la última década, una nueva visión del TDAH ha superado las explicaciones centradas en los tres déficits primarios citados anteriormente (inatención, impulsividad e hiperactividad) ofreciendo un enfoque más comprensivo del trastorno y explicando mejor toda la sintomatología que se observa en el TDAH, incluyendo las alteraciones lingüísticas. Las teorías más actuales explican este trastorno como un déficit en la función ejecutiva justificando el amplio espectro de síntomas presentes en él, integrando datos neurobiológicos, cognitivos y conductuales. De entre las teorías surgidas en los últimos años, merece destacarse, en nuestra opinión, la del Dr. Russel Barkley (1997 a). Este investigador define el TDAH como un déficit en el control inhibitorio, que constituye el primer acto autorregulatorio de la conducta. El déficit de control inhibitorio incide a su vez de forma negativa en cuatro funciones neuropsicológicas que dependen de la inhibición conductual para su efectiva ejecución: a) memoria de trabajo; b) autorregulación de la motiva- 
ción y del afecto; c) internalización del lenguaje; y d) procesos de análisis y síntesis.

El modelo de Barkley no considera las dificultades atencionales como el síntoma primario del TDAH. Éstas son una consecuencia de la alteración que la escasa inhibición conductual y el bajo control de la interferencia crean en la autorregulación o control ejecutivo del comportamiento. De hecho las manifestaciones de desatención de los niños hiperactivos no se manifiestan en situaciones y tareas que requieren atención selectiva sino en aquellas que plantean una alta exigencia autorregulatoria de los recursos atencionales. No obstante, el papel otorgado a la inhibición por Barkley, como un constructo superior en la jerarquía de las funciones ejecutivas, difiere del de otros autores interesados en las patologías del sistema ejecutivo. Así, Ozonoff y colaboradores (Ozonoff, Strayer, McMahon y Fillow, 1994) definen la función ejecutiva como el constructo cognitivo utilizado para desarrollar conductas dirigidas a una meta, orientadas hacia el futuro, que se consideran mediadas por los lóbulos frontales. Incluyen la planificación, inhibición de respuestas prepotentes, flexibilidad, búsqueda organizada y memoria de trabajo. Es muy probable que todas estas funciones ejerzan una labor equilibradora unas sobre otras, de forma que la disfunción de una de ellas altere las demás. Pero, tanto desde una perspectiva más piramidal como desde una perspectiva más conexionista, el déficit en la función ejecutiva tiene un papel clave en la formulación actual del TDAH, cobrando especial importancia el papel explicativo que se otorga a la memoria de trabajo en las dificultades que observamos en este trastorno (McInnes, Humphries, Hogg-Johnson y Tannock, 2003). A este respecto, no debemos olvidar el papel de la memoria de trabajo,-que se ha descrito como «un espacio mental de trabajo» para llevar a cabo el procesamiento de la información-, en la comprensión del lenguaje y en el procesamiento del discurso (Kintsch, 1998; Zwaan y Radvansky, 1998).

Algunos trabajos han puesto de relieve que los niños con TDAH, en conjunto, tiene más dificultades, que los niños que no sufren este problema, para realizar las tareas clásicas de morfología, semántica o metafonología que tienen más exigencia en memoria de trabajo y, por tanto, más control ejecutivo. Por ejemplo, tienen más dificultades para realizar tareas que exigen establecer analogías que para realizar aquellas que implican únicamente conocimiento del vocabulario (Ygual, Miranda y Cervera, 2000). Tienen más dificultades para comparar segmentos fonológicos comunes a dos palabras que para aislar un segmento en una sola palabra (Miranda, Ygual, Mulas, Roselló y Bó, 2002). No se trata de dificultades en la capacidad estructural de memoria («span» de memoria) (McInnes, 
Humphries, Hogg-Johnson y Tannock, 2003) sino en la habilidad para mantener activa en la mente la información relevante para una tarea durante el procesamiento de la información. Aún más, los déficits en función ejecutiva y, concretamente, en memoria de trabajo marcan de forma significativa las habilidades comunicativas de los niños con TDAH y de forma particular aquellos actos lingüísticos de más exigencia cognitiva como, por ejemplo, el relato.

\section{La investigación sobre las habilidades narrativas de los niños con TDAH}

En los primeros trabajos que se ocuparon del lenguaje de los niños con TDAH, coincidiendo con la preocupación por sus dificultades de índole cognitiva, ya se observaron los problemas que presentaban estos niños en contextos lingüísticamente complejos como la narración. Los trabajos de Sydney S. Zentall (1988) figuran entre los pioneros en el estudio de las habilidades narrativas de los niños con TDAH. Esta investigadora observó la actuación de 22 niños hiperactivos (20 chicos y dos chicas), comparándola con niños normales. Los sometió a varias condiciones experimentales, unas en las que se inducía lenguaje y otras no. Las primeras eran las siguientes: a) Contar una historia, un incidente real elegido por el propio el niño; b) Contar una historia a partir de cuatro palabras clave dadas; c) Contar una historia a partir de unos dibujos y d) Volver a contar una historia que habían oído previamente. Como se puede observar, la diferencia entre las distintas situaciones experimentales presentadas estriba en el grado de estructuración de estímulo que elicita el relato, desde un estímulo no estructurado (a) hasta un estímulo altamente estructurado que ofrece un modelo completo a reproducir (d). La condición experimental en la que no se inducía lenguaje consistió en la realización de 10 ítems elegidos del MFFT-20. El análisis de los resultados señaló que el déficit en la producción verbal de los niños hiperactivos se manifestó en las tareas de contar una historia con un estímulo completo (volver a contar una historia que se ha escuchado previamente), así como en la de contar una historia sin un estímulo, sin un modelo.

Curiosamente, los niños hiperactivos fueron más habladores que sus iguales cuando no se les pedía que hablasen (tarea MFFT-20) pero hablaron menos cuando se les solicitaba que contasen una historia. Hablaron menos en las tareas verbales que requerían una organización interna en la respuesta ya que este efecto no se dio en las tareas en las que el 
estímulo estaba visualmente presente y secuenciado (contar una historia a partir de dibujos). El hecho de que los niños hiperactivos utilizasen menos palabras ante las tareas de contar una historia, podía indicar que las deficiencias de producción encontradas eran atribuibles a los requerimientos de organización y planificación que exigían este tipo de tareas. Según la interpretación de Zentall, las dificultades de memoria no habían contribuido a las deficiencias de producción.

Posteriormente, Tannock, Purvis y Schachar (1993) analizaron el tipo de errores que los niños con TDAH cometían durante la narración de historias siguiendo la hipótesis de que la frecuencia y tipo de errores podían ser indicadores de los procesos ejecutivos subyacentes de organización y control que distinguían a los niños con TDAH de los niños normales. Trabajaron con 30 niños TDAH y 30 normales, de 7 a 11 años, y utilizaron como estímulo dos cuentos populares, desconocidos para los niños, grabados en audio. Midieron dos aspectos: comprensión y producción. El análisis de la comprensión se realizó a través de las respuestas a dos tipos de preguntas, unas sobre los hechos contenidos en las historias y otras sobre información inferencial. Se estudió la producción a través del tipo de errores que cometieron en la narración. Los resultados no mostraron en los niños TDAH dificultades en la comprensión y extracción de las ideas principales de las historias, pero se observaron problemas en la producción en cuanto a la organización, exactitud y cohesión de las historias contadas, así como en la cantidad de información aportada, que era menor que la del grupo normal.

Además, los niños con TDAH cometieron más errores de secuenciación y más errores de cohesión. Se observaron más errores en la interpretación de la información y el uso inapropiado de pronombres. Estos errores pueden mostrar una ruptura en la comprensión de la historia, aunque Tannock, Purvis y Schachar no lo interpretaron así. Dado que no se encontraron diferencias en las medidas de comprensión entre el grupo TDAH y el normal, los interpretaron como fallos en el control de la exactitud de la información que reflejaban, por tanto, un déficit en los procesos de control ejecutivo. Asimismo estos investigadores manifestaron que no había evidencia de déficit en la memoria verbal o en la capacidad de memoria per se. Estos hallazgos fueron apoyados por una investigación posterior (Purvis y Tannock, 1997), en la que los niños con TDAH eran comparados con niños con dificultades lectoras. En este trabajo se reafirmaba la idea de que los niños con TDAH son capaces de identificar las ideas relevantes de la narración. Sin embargo, cuando se les pide que relaten la historia, cuentan menos ideas relevantes y tienen dificultad para organizar todos los sucesos de forma coherente y con cohesión. 


\section{Alteraciones del relato: los niños con TDAH}

Las dificultades de expresión en una tarea compleja, como la narración de historias, quedaban suficientemente probadas. Sin embargo, sorprende, en cierta medida, la ambigüedad en torno al «input» de la información, a la comprensión de la historia durante la escucha. Los niños con TDAH tienen problemas en un grupo importante de procesos que están implicados en la comprensión de las narraciones: deficiencias en atención sostenida, que determinan que dirijan menos esfuerzo hacia las demandas de la tarea; menos capacidad en memoria de trabajo, y problemas inhibitorios que pueden interferir en la comprensión de la historia. Por consiguiente, cabría preguntarse si es posible que la comprensión de la información no estuviera alterada en los niños con TDAH. Enfocado de otra manera: ¿las dificultades de los niños con TDAH se centran en la cantidad de recursos cognitivos empleados para la comprensión de la historia o se centran en el reparto de los recursos disponibles durante la narración? Por ejemplo, las dificultades para dedicar suficiente esfuerzo o mantenimiento de la atención es probable que disminuyan la cantidad de recursos que se aplicarán en la tarea. Así mismo, el funcionamiento de la memoria de trabajo puede tener mayor impacto en el reparto de los recursos que en la cantidad de los mismos. Una memoria de trabajo limitada fuerza un reparto diferencial de la atención, creando conexiones inmediatas entre los sucesos de la historia a expensas de conexiones causales que requieren recuperar información anterior. De la misma forma, los fallos para suprimir la activación de nudos o conexiones irrelevantes afectarían negativamente a la información que se utiliza para crear una representación de la historia más elaborada.

Lorch, Diener, Sánchez, Milich, Welsh y Van den Broek (1999) trataron de dar respuesta a estas cuestiones, afrontando el análisis de las narraciones en los niños con TDAH desde otro punto de vista. Utilizaron los mismos estímulos que Tannock et al. (1993) pero emplearon un modelo de análisis distinto: el análisis de la red causal (Trabasso, Secco y van den Broek, 1.984; Trabasso y van den Broek, 1985). Los resultados que obtuvieron mostraban que, en general, los niños con TDAH presentan déficits tanto en la cantidad de recursos cognitivos que dedican a la comprensión de la historia como en la forma en que distribuyen esos recursos.

No obstante, observaron diferencias moduladas tanto por el género como por el nivel de inteligencia. La comprensión de la historia de las chicas mostró patrones diferentes dependiendo del nivel de inteligencia. Las chicas con alto nivel de inteligencia no mostraron déficit en la realización de la tarea; parecían tener habilidades cognitivas que les permitían compensar algunos déficits asociados al estatus de TDAH. Por el contrario, 
las que tenían más bajo nivel de inteligencia tuvieron un rendimiento peor y no fueron sensibles a la estructura causal de la historia. Algunos trabajos han diferenciado dos subtipos entre las chicas con diagnóstico de TDAH dependiendo de su nivel de inteligencia. Las chicas TDAH con nivel bajo de inteligencia parecen mostrar más problemas cognitivos que otros grupos con similar nivel de inteligencia (Gaub y Carlson, 1997; James y Taylor, 1990).

Los chicos reflejaron un patrón diferente al de las chicas. En conjunto contaron menos sobre la historia y el efecto de las conexiones causales fue menor que los del grupo de comparación de alta inteligencia y similar al grupo de comparación de baja inteligencia. El hecho de que la actuación de los chicos con TDAH no estuviera modulada por la inteligencia sugería que sus dificultades en la comprensión de la historia podían estar más asociadas a su sintomatología de TDAH que a déficits cognitivos específicos, según Lorch et al. (1999). Aspectos como las dificultades para mantener la atención pueden provocar un procesamiento menos consistente de los sucesos de la historia, creando huecos en la representación resultante. Además, los déficits en la memoria de trabajo pueden interferir en la habilidad de los chicos con TDAH para desarrollar una representación coherente de la historia, porque son menos capaces de mantener la activación de los antecedentes mientras ellos procesan nueva información. Una tercera posibilidad es que los problemas de control inhibitorio puedan impedir la habilidad «on-line» para suprimir la activación de la información que no es relevante para establecer conexiones correctas. Como consecuencia, los recursos cognitivos no están focalizados en la información que es necesaria para construir en la memoria una representación de la historia coherente y elaborada. En la misma línea se encuentran las conclusiones del trabajo de Milch-Reich, Campbell, Pelham, Connelly y Geva (1999) que también evidenció que algunas de las dificultades que se observan en los niños con TDAH pueden ocurrir durante el procesamiento «on-line» de las historias. Su trabajo fue realizado en niños con TDAH, en edades de la escuela elemental, que fueron comparados con niños normales. Se les pidió que contasen una historia representada por una serie de dibujos. Fue utilizado también el método de análisis de la red causal.

En otro trabajo del grupo de Lorch (Lorch, Sánchez, Van den Broek, Milich, Murphy, Lorch Jr. y Welsh, 1999) se evaluó la capacidad de niños, entre cuatro y seis años, para captar la estructura de la historia utilizando como estímulo series de televisión, concretamente Barrio Sésamo. Ellos partían del hecho de que el visionado de series de televisión ofrecía una ventaja: el alto grado de atención que los niños con TDAH 
mostraban frente a la televisión. Esto posibilitaría la evaluación más aislada de otros aspectos como la memoria. Se aplicaron dos condiciones de visionado de la historia: televisión con presencia de juguetes como efecto distractor y sólo televisión. Los resultados informaron de que los niños pequeños con TDAH pueden crear una representación, similar a la de su grupo de comparación, de los hechos de una historia sencilla bajo condiciones óptimas de visión. Sin embargo, diferían en cuanto a las conexiones causales entre los sucesos, especialmente en presencia de actividades distractoras. Por tanto, parecía que las dificultades en el control inhibitorio también influían negativamente en la capacidad de procesamiento «on line». En un trabajo reciente (Lorch, Eastham, Milich, et al., 2004), concluyen que las dificultades de los niños TDAH para responder a preguntas que implican relaciones causales, sobre una historia que han visionado, obedecen a su menor grado de implicación cognitiva. Esta dificultad está relacionada con un menor tiempo de mantenimiento de la atención, mermando su capacidad para establecer vínculos causales entre acontecimientos relativamente distantes en el tiempo. Las dificultades atencionales se producen, también en este caso, sólo cuando están presentes los juguetes, por tanto, parece implicado el sistema inhibitorio.

Cohen, Vallance, Barwick et al. (2000) evaluaron tanto habilidades narrativas como habilidades estructurales del lenguaje en niños con TDAH más dificultades de lenguaje, y en niños con dificultades de lenguaje únicamente, sin TDAH. Ellas, sin embargo, no encontraron diferencias significativas entre ambos grupos. Aunque observaron como la memoria de trabajo verbal incide en el procesamiento de los niños con TDAH, consideraron que sus problemas están más asociados a las dificultades del lenguaje que al TDAH, ya que, también piensan que el déficit en la memoria auditiva verbal justifica los problemas pragmáticos que tienen los niños con dificultades de lenguaje. Además, el déficit en niveles estructurales del lenguaje está asociado con una inmadurez en el razonamiento cognitivo social, que es un componente de la pragmática comunicativa.

Recientemente, hemos estudiado la narración de los niños con TDAH en lengua castellana con un doble objetivo: observar las diferencias de los niños con TDAH con respecto a los que no padecen este trastorno y las diferencias entre los subtipos más frecuentes del trastorno, el subtipo predominantemente inatento y el combinado (Ygual, 2003). Participaron en el trabajo 56 niños, 28 con un diagnóstico de TDAH y 28 normales, con edades comprendidas entre 5 años y nueve meses y 12 años. Se elicitó la narración a partir de la escucha de una historia (retelling), utilizando únicamente el estímulo auditivo (sin presencia de dibujos que actuasen 
como organizador externo). El niño escucha una historia previamente grabada y luego la tiene que contar. Se utilizó una historia compleja, tenía cuatro episodios. Seguía la estructura de la Gramática de la Historia de Stein y Glenn (1979), según la cual el protagonista se ve envuelto en una situación conflictiva, debe formular un plan y hacer múltiples intentos para resolverlo. Al final el plan es realizado satisfactoriamente con unas consecuencias positivas.

Todos los episodios que constituyen la historia, tienen una estructura lógica idéntica y constituyen una unidad de contenido en sí mismos. Cada episodio está formado por seis componentes:

1. Planteamiento informativo o marco. A este componente pertenecen todas las frases o, para ser estrictos, todas las secuencias o unidades de contenido que hacen referencia a escenarios, personajes, localizaciones o contextos habituales y estados.

2. Suceso inicial. Acciones, hechos, cambios en el entorno físico o en la percepción interna que un personaje tiene de un acontecimiento que suponen un obstáculo físico, un dilema moral, un acontecimiento ambiental o un problema personal.

3. Respuesta interna. El personaje responde a la situación e idea un plan. A este componente responden todas las secuencias que hacen referencia a emociones de un personaje, formulación de objetivos, deseos, intenciones o pensamientos que llevan a un plan secuencial.

4. Intento-acción. Los intentos o acciones que hace el personaje, o personajes protagonistas, para resolver la situación o conseguir su objetivo.

5. Consecuencia directa. Acciones, sucesos naturales o estados finales que representan la consecución o no del objetivo propuesto por el personaje.

6. Reacción. Cómo el personaje siente, piensa o actúa en relación a la consecuencia directa.

Se estudió la coherencia de la narración generada por los niños a través del número de episodios que narraban completos y el tipo de componentes que aparecían en el relato. Asimismo, se analizó el grado de comprensión de la historia utilizando dos series de preguntas, una serie referente a datos concretos de la historia y otra de tipo inferencial.

Nuestros resultados confirman que las habilidades narrativas de los niños con TDAH son peores que las de los niños que no tienen este problema. Cuando se les pide que cuenten historias, previamente oídas, cuentan historias significativamente más cortas, ofrecen mucha menos 
información acerca de ellas, y las narraciones son más desorganizadas y menos coherentes. Si atendemos al tipo de información relatada, aportan menos información de todos los tipos de secuencias de la gramática de la historia. Relatan menos información sobre los personajes, menos información sobre aspectos descriptivos generales como localización, tiempo, etc. Aportan menos información sobre los obstáculos o problemas con que se encuentran los personajes y que son causa de su actuación y menos información acerca de cómo superan esos obstáculos y los planes de acción que aplican para ello. Facilitan menos datos acerca de las acciones efectuadas por los personajes principales y sobre las consecuencias de las mismas. Asimismo, relatan menos datos sobre las respuestas emocionales, metas, pensamientos y deseos de los personajes.

En cuanto a la comprensión de la historia, los niños TDAH cometieron más errores al ser preguntados sobre la información general y los detalles concretos sobre las historias. De igual forma, tuvieron más problemas para establecer relaciones entre la información, relaciones de causalidad, consecuencias etc. Por tanto nuestros datos parecen indicar que las dificultades en las narraciones comienzan ya en la fase de comprensión.

La narración de historias es una prueba compleja y exigente para la evaluación del lenguaje porque requiere un buen conocimiento de las estructuras básicas del mismo y plantea demandas importantes en planificación interna, organización y control simultáneo de la información. Sin duda, el déficit en el dominio de las estructuras básicas del lenguaje, que se observa en muchos de los niños con TDAH, incide negativamente en las narraciones. Pero en el mal rendimiento ante tareas de narración de historias, las dificultades en el funcionamiento ejecutivo del que dependen las tareas de planificación, organización y control de la información, posiblemente, sean un factor crucial.

Cuando los niños relatan secuencias o unidades de comunicación referidas a cómo los personajes superan un obstáculo o resuelven un problema, deben de hacer un uso especial de la memoria de trabajo que les proporcione una visión retrospectiva (datos sobre los sucesos anteriores que han motivado el problema y/o la forma de actuar de los personajes) y una previsión (datos sobre la conducta anticipatoria y sobre el propósito de la acción), realizando, por tanto, un análisis y una síntesis de la conducta. Cuando relatan los efectos de la conducta realizada por los personajes principales, que superan el problema o complican más la situación, deben establecer contingencias entre las acciones y los resultados de las mismas. Al describir secuencias referidas a las respuestas emocionales, metas, pensamientos y deseos de los personajes, son requeridos aspectos 
de regulación de la emoción y marcadores del lenguaje interno que indican procesos de regulación sobre la conducta dirigida a meta. Los problemas en la narración de historias reflejan dificultades en el manejo de todos estos aspectos relacionados con la función ejecutiva. Estas son dificultades que se observan también en la vida cotidiana de los niños con TDAH.

El tipo de errores al contar historias también puede relacionarse con el funcionamiento ejecutivo. Los errores consistentes en añadir una idea a la historia que no figuraba en la versión original, se relacionan con problemas en la función de inhibición. Por ejemplo, uno de los niños en lugar de comenzar a contar la historia de «Los tres hermanos que eran marineros», comenzó diciendo «eran tres cerditos marineros», probablemente por la similitud del término «tres». Los errores en la secuenciación de la historia indican problemas con la memoria de trabajo y el procesamiento temporal. Los errores en los que se interpreta de forma incorrecta el significado (mala interpretación y referencias ambiguas) dependen del control de la exactitud de la información. Los errores de repetición de unidades de información se unen a problemas de autocontrol y de seguimiento de la historia. Cuando un personaje, objeto o suceso es sustituido por otro de forma inapropiada (error de sustitución) se relaciona con un problema en la recuperación de la información de la memoria a largo plazo.

Recapitulando, los métodos que han utilizado los diversos trabajos para el análisis de narraciones han sido distintos, pero los resultados han sido convergentes: los niños con TDAH tienen una especial dificultad en la narración de historias que se relaciona con dificultades en el funcionamiento ejecutivo. Tannock, Purvis y Schachar (1993) analizaron las habilidades narrativas observando el tipo de errores de producción. Los niños con TDAH cometieron más errores de secuenciación y más errores en la interpretación de la información. Purvis y Tannock (1997) posteriormente evaluaron habilidades semánticas (en comprensión y expresión) y habilidades narrativas. En este último aspecto, los resultados fueron idénticos. En cambio, tanto en un trabajo como en otro, afirman no encontrar, en los niños con TDAH, dificultades para comprender las ideas relevantes de la historia. Sin embargo, en un trabajo reciente (McInnes, Humphries, Hogg-Johnson y Tannock, 2003) concluyen que los niños con TDAH tienen dificultades en comprensión auditiva relacionadas con la memoria de trabajo. Lorch et al. (1999) sí encontraron dificultades de comprensión en las narraciones utilizando el modelo de análisis de red causal y en nuestro trabajo también las hemos observado desde el modelo de análisis de gramática de la historia. 
Sin embargo, es posible que no todos los niños con TDAH tengan las mismas dificultades de comprensión. Nosotros hemos observado diferencias entre el subtipo combinado y el inatento, de manera que este último tiene más dificultades en aspectos de comprensión aunque las dificultades de producción son similares. Este hallazgo parece coherente con la información de que disponemos sobre el funcionamiento cognitivo de los niños TDAH/I, que parecen tener más déficits en tareas de atención auditiva (Barkley, 1997; Bedi, Halperin y Sharma, 1994; Carella, 1998). Por tanto, ambos subtipos, TDAH/I y TDAH/C, tienen dificultades en la narración pero es posible que sea por razones distintas. Mientras que el grupo TDAH/I tiene menos recursos cognitivos disponibles para la realización de una tarea como las narraciones, el grupo TDAH/C falla en reparto y control de esos recursos.

En suma, el estudio de las habilidades narrativas de los niños con TDAH ha aportado una valiosa información sobre el procesamiento de la información en estos niños. Las dificultades que presentan se pueden producir en varios estadios del procesamiento: las dificultades atencionales que pueden influir interfiriendo en la entrada de la información; las dificultades en la memoria operativa los hace menos eficaces en el reparto y control de recursos durante la realización de la tarea, lo cual se traduce en dificultades en la evocación de sucesos, más errores en el establecimiento de conexiones causales de los mismos que estaría interferida por la no desactivación de información irrelevante para la narración. La memoria verbal, concretamente, es un elemento clave en las funciones ejecutivas de los niños con TDAH. Hallazgos consistentes en la literatura indican que la memoria de trabajo tiene una base lingüística incluso cuando también está implicada la información no verbal (Denckla, 1996a, b).

Por último, también se ha observado en el discurso (aunque este aspecto no ha sido muy estudiado) dificultades en la selección y manejo de las estructuras lingüisticas de base produciendo más errores de cohesión.

Es importante señalar que esta actuación de los niños con TDAH es perfectamente generalizable a situaciones de la vida real, el lector puede pensar, por ejemplo, en el seguimiento de una explicación del profesor en clase y su posterior representación en memoria. Las dificultades en realizar una codificación consistente y en la utilización de las relaciones causales entre las ideas conlleva, también, numerosas implicaciones en la vida diaria de tipo social y académico. Algunos estudios demuestran que los niños con TDAH experimentan dificultades para interpretar las acciones de los otros niños y para realizar conexiones entre sus propias acciones y las consecuencias de las mismas (Milch-Reich, Campbell, Pel- 
ham, et al., 1999). En la escuela, a medida que los niños con TDAH crecen y avanzan a cursos superiores, se ven obligados a enfrentarse a tareas que requieren la comprensión y producción estructurada de relatos. El desarrollo representacional, la construcción de las bases del conocimiento y las estrategias de solución de problemas se apoyan, entre otros procesos organizativos, en la competencia para la comprensión y producción narrativa. Son muchos los conocimientos sobre el funcionamiento cognitivo de los niños con TDAH que nos han aportado las investigaciones sobre sus habilidades narrativas pero es, sin duda, un tema apasionante sobre el que hay que seguir profundizando.

\section{Referencias bibliográficas}

BARKLEy, R. A. (1997a): Behavioral Inhibition, Sustained Attention, and Executive Functions: Constructing a Unifying Theory of ADHD. Psychological Bulletin, 121, 65-94.

BARKLEY, R. A. (1997b): ADHD and the Nature of Self-control. New York: The Guilford Press.

Bedi, G.C., Halperin, J.M., y Sharma, V. (1994): Investigation of Modality Specific Distractibility in Children. International Journal of Neurosciencie, 74, 79-85.

Carella, S.E. (1998): The Cognitve Profiles of the Inattentive and Hyperactive/Impulsive Subtipes of Attention Deficit-hyperactivity Disorder. Dissertation Abstracts International Section B: Sciences and Engigeering, 58, 6229.

Cohen, N-J; VAllance, D-D.; Barwick, M-A.;Im, N.; Menna, R.; HorodezKy, N.; IsaAcson, L. (2.000): The Interface between ADHD and Language Impairment: An Examination of Language, Achievement and Cognitive Processing. Journal of Child Psychology and Psychiatry, 41, 353-362.

GaUb, M. y CARLSON, C.L. (1997): Gender Differens in ADHD: A Meta-analysis and Critical Review. Journal of American Academy of Child and Adolescent Psychiatry, 36, 1036-1046.

James, A. y TAYlor, E. (1990): Sex Differences in the Hyperkinetic Syndrome of Childhood. Journal of Child Psychology and Psychiatry, 30, 383-387.

Kintsch, W. (1998): Comprehension. A Paradigm for Cognition. Cambridge, UK: Cambridge University Press.

LorCh, E.; Diener, M.;SÁNChez, R.; Milich, R.; Welsh, R. y VAN DEN Broek, P. (1999): The Effects of Story Structure on the Recall of Stories in Children with Attention Deficit Hyperactivity Disorder. Journal of Educational Psychology, 91, 273-283.

Lorch, E.; SÁnchez, R.; VAN DEN BRoek, P.; Milich, R.; MurPhy, E.; LorCh JR. R. y Welsh, R. (1999): The Relation of Story Structure Properties to Recall of Television Stories in Young Children with Attention Deficit Hyperactivity Disorder and Nonreferred Peers. Journal of Abnormal Child Psychology, 27, 293-309.

Lorch, E.P., Eastham, D., Milich, R., Lemberger, C.C., Sanchez, R.P., Welhs, R., van DEN Broek., P. (2004): Difficulties in Comprehending Causal Relations Among Children with ADHD: The Role of Cognitive Engagement. Journal of Abnormal Psychology, 113, 56-63.

Mcinnes, A., Humphries, T., HogG-Johnson, S. y Tannock, R. (2003): Listening Comprehension and Working Memory are Impaired in Attention-deficit Hyperactivity Di- 


\section{Alteraciones del relato: los niños con TDAH}

sorder Irrespective of Language Impairment. Journal of Abnormal Child Psychology, $31,427-443$.

McInnes, A., Humphries, T., Hogg-Johnson, S. y TAnnock, R. (2003): Listening Comprehension and Working Memory are Impaired in Attention Deficit Hyperactivity Disorder Irrespective of Language Impairment. Journal of Abnormal Child Psychology, $31,427-443$

Milch-Reich, S., Campbell, S.B., Pelham, W. E., Jr., Connelly, L.M. y Geva, D. (1999): Developmental and Individual Differences in Children's on-line Representations of Dynamic Social Events. Child Development, 70, 413-431.

Miranda, A.; Roselló, B. y Soriano, M. (1998): Estudiantes con deficiencias atencionales. Valencia. Ed. Promolibro.

Miranda, A.; Ygual, A. y Rosel, J. (2004): Complejidad gramatical y mecanismos de cohesión en la pragmática comunicativa de los niños con trastorno por déficit de atención con hiperactividad. Revista de Neurología,38 (S 1), 111-116.

Miranda, A.; Ygual, A.; Mulas, F.; Roselló, B. y Bó, R. (2002): Procesamiento fonológico en niños con trastorno por déficit de atención con hiperactividad: ¿Es eficaz el metilfenidato?. Revista de Neurología,34(S 1),115-121.

Ozonoff, S., Strayer, D.L., McMahon, W.M. y Fillow, F (1994): Executive Function Abilities in Autism and Tourette Syndrome: An Information Processing Approach. Journal of Child Psychology and Psychiatry, 35, 1015-1032.

Purvis, K.L. y TANNOCK, R. (1997): Language Abilities in Children with Attention Deficit Hyperactivity Disorder, Reading Disabilities and Normal Control. Journal of Abnormal Child Psychology, 25, 133-144.

Stein, N.L. y GLENN, C.G. (1979): An Analysis of Story Comprensión in Elementary School Children. In R.O. Freedle (Ed.), New Directions in Discourse Processing (Vol. 2, pp. 53-119). Norwood, NJ: Ablex.

Tannock, R., Purvis, K y Schachar, R. (1993): Narrative Abilities in Children with Attention Deficit Hyperactivity Disorder and Normal Peers. Journal of Abnormal Child Psychology, 21, 103-117.

Trabasso, T., Secco, T. y van Den Broek, P.W. (1984): Causal Cohesión and Story Coherence. In H. Mandl, N.L. Stein y T. Trabaos (Eds.). Learning and Comprehension of Text (pp. 83-111). Hillsdale, NJ: Erlbaum.

Trabasso, T., y VAN DEN BROEK, P. (1985): Causal Thinking and the Representation of Narrative Events. Journal of Memory and Language, 24, 612-630.

YGuAL, A. (2003): Problemas de lenguaje en estudiantes con déficit atencional. [Tesis doctorall. Valencia: Universidad de Valencia.

Ygual, A.; Miranda, A. y Cervera, J. (2000): Dificultades en las dimensiones de forma y contenido del lenguaje en los niños con trastorno por déficit de atención con hiperactividad. Revista de Neurología Clínica, 1, 193-202.

Zentall, S. (1988): Production Deficiencies in Elicited Language but not in the Spontaneus Verbalizations of Hyperactive Children. Journal of Abnormal Child Psychology, 16, 657-673.

ZwaAn, R. y RAdVANsky, G.A. (1998): Situation Models in Language Comprehension and Memory. Psychological Bulletin, 123, 162-185. 